\title{
Polimorfismo SNP-43 del gen de Calpaína-10 en individuos con enfermedad coronaria y controles
}

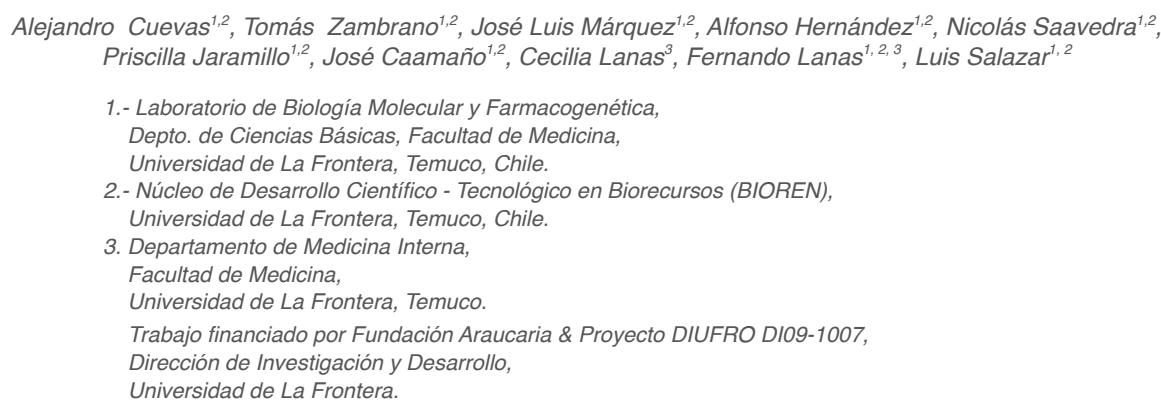

\section{Resumen:}

La Diabetes mellitus tipo 2 (DMT2) es un reconocido factor de riesgo cardiovascular que ha mostrado asociación con aterosclerosis subclínica; lo cual podría ser explicado por la presencia de una base genética común entre ambas patologías. Varios polimorfismos del gen de calpaína-10 (CAPN10) han sido asociados con insulino resistencia y DMT2, sin embargo, existe escasa evidencia de su relación con enfermedad coronaria. El objetivo del presente estudio fue evaluar la posible asociación de la variante SNP-43 de CAPN1O con el desarrollo de enfermedad coronaria en individuos del sur de Chile.

Métodos: Fueron incluidos en este estudio, 218 pacientes adultos no relacionados con enfermedad arterial coro- naria (EAC) confirmada mediante angiografía (estenosis $>70 \%$ ) y 194 individuos controles. La variante genética UCSNP-43 fue estudiada por PCR-RFLP.

Resultados: No se observaron diferencias significativas entre las frecuencias genotípicas de la variante UCSNP-43 en pacientes con EAC (GG: $51.4 \%$; GA: 42.7 $\%$; AA: $5.9 \%$ ) y controles (GG: $59.6 \%$; GA: $35.1 \%$; AA: $5.2 \%, \mathrm{p}=0.228$ ). Similarmente, no hubo diferencias significativas entre las frecuencias alélicas entre pacientes con EAC y controles $(\mathrm{p}=0.200)$. La Odds Ratio fue de 1.17 (I.C. 95\%: 0.50-2.72), confirmando la ausencia reasociación.

Conclusión: Nuestros datos sugieren que la variante UCSNP-43 del gen CAPN10 no está asociada a la presencia de enfermedad arterial coronaria en la población investigada.

\section{SNP43 polymorphism of Calpain-10 with coronary artery \\ gene in Southern Chilean subjects disease and controls}

Background: Diabetes mellitus type 2 (DMT2) is a well know cardiovascular risk factor and has been related to subclinic atherosclerosis, which might be explained by the presence of a common genetic basis in both diseases. Several polymorphisms of the calpain-10 gene (CAPN10) have been associated with insulin resistance and DMT; nevertheless, there is insufficient evidence about their relation with coro- nary artery disease (CAD). Thus, in the present study we investigated the possible association between the SNP-43 variant of CAPN10 and the presence of CAD in Chilean subjects.

Methods: A total of 218 unrelated patients with diagnosis of CAD confirmed by angiography (33-74 years old) and 194 healthy controls (30 - 68 years old) were included in this study. The SNP43 variant of the 
CAPN10 gene was evaluated by PCR-RFLP. Results: The genotype distribution for SNP43 variant of CAPN10 gene in CAD patients (GG: $51.4 \%$; GA: $42.7 \%$; AA: $5.9 \%$ ) and controls (GG: 59.6\%; GA: $35.1 \%$; AA: $5.2 \%)$ was similar $(\mathrm{P}=0.228)$. Similarly, the allelic frequency was no different $(P=0.200)$. The $\mathrm{OR}$ for $\mathrm{CAD}$ related to $\mathrm{AA}$ homozygous genotype was

\section{Introducción:}

Diabetes mellitus tipo 2 (DTM2) es uno de los más potentes factores de riesgo para enfermedad arterial coronaria (EAC). Se ha reportado que ésta patología aumenta el riesgo relativo de enfermedad cardiovascular 2 a 4 veces, respecto a población no diabética ${ }^{1}$. Además, individuos diabéticos tienen una mayor incidencia de las formas clínicas más severas de eventos coronarios, como infarto agudo al miocardio y muerte súbita, así como una mayor tasa de mortalidad posterior a un infarto agudo al miocardio, respecto a individuos sin ésta patología ${ }^{1}$. Haffner et al., demostraron que individuos no diabéticos, de edad media y con enfermedad coronaria preexistente, tienen una incidencia a los 7 años de infarto agudo al miocardio similar a la de individuos diabéticos sin enfermedad coronaria, sugiriendo que la diabetes confiere el mismo grado de riesgo que una enfermedad coronaria preexistente ${ }^{2}$. Por otro lado, la historia familiar de diabetes es un predictor independiente de engrosamiento medio-intimal de las arterias carótidas ${ }^{3}$, medida indirecta utilizada para valorar aterosclerosis subclínica.

Tanto DMT2 como EAC tienen un fuerte componente genético en su etiología y factores genéticos que predispongan a una mayor susceptibilidad de padecer DMT2, pudiesen incidir sobre el riesgo de padecer EAC. Al respecto, CAPN10 fue el primer gen identificado por clonaje posicional, en el loci de susceptibilidad para DMT2, denominado NIDDM1 (Non-insulin dependent diabetes mellitus 1$)^{4}$ El gen CAPN10, codifica una proteasa de cisteína no lisosomal, la calpaína 10 , cuya función no es completamente conocida. Se ha demostrado que la calpaína-10 está envuelta en el mecanismo de secreción de la insulina ${ }^{5}$, en apoptosis de las células $\beta$ pancreáticas ${ }^{6} \mathrm{y}$ en la utilización oxidativa de la glucosa por el músculo esquelético, entre otros hallazgos.

Estudios en varias poblaciones han asociado variantes del gen CAPN10 a patologías como el síndrome de ovario poliquístico ${ }^{7}$ y DMT2 ${ }^{8-10}$, cuya etiología está
$1.17(95 \%$ C.I. $=0.50-2.72)$, confirming the absence of association.

Conclusion: These findings suggest that the SNP43 polymorphism of the CAPN1O gene is not associated to $\mathrm{CAD}$ in the studied individuals.

Keywords: Coronary artery disease; Gene polymorphism; CAPN10.

asociada a una secreción defectuosa de insulina. Entre estas variantes genéticas, principalmente tres han sido asociadas con un aumento del riesgo de padecer DMT2 de hasta un 14\%: los SNPs -43,-19 y -63, todos ubicados en regiones no codificantes de CAPN10 (intrones 3, 6 y 13 , respectivamente).

Recientemente, estas variantes fueron asociadas con aterosclerosis subclínica y factores que determinan un fenotipo pre-diabéticos en población México-americana no diabética ${ }^{11}$, sugiriendo que DMT2 y EAC pueden tener bases genéticas comunes. Sin embargo, estos hallazgos no han sido replicados en otras poblaciones. Así, el objetivo de este estudio fue evaluar la posible asociación entre el polimorfismo SNP-43 del gen CAPN10 y EAC en individuos chilenos del sur de nuestro país.

\section{Métodos:}

\section{Sujetos}

Este estudio, de tipo casos y controles, investigó la frecuencia del polimorfismo UCSNP-43 del gen CAPN10 en un total de 412 individuos no relacionados, todos pertenecientes a la Región de La Araucanía, Chile.

El grupo casos quedó formado por 218 individuos con diagnóstico de enfermedad arterial coronaria (historia y ECG de infarto al miocardio o enfermedad coronaria comprobada por angiografía con estenosis superior al 70\%), provenientes del Hospital Hernán Henríquez Aravena de la ciudad de Temuco. El grupo control correspondió a 194 individuos no relacionados, sin signos de enfermedad cardiovascular al momento de participar de este estudio. Para ninguno de los grupos evaluados existió preselección según los niveles séricos de lípidos. De cada individuo se obtuvieron datos de presión arterial sistólica y diastólica, además del antecedente de si recibían algún tipo de tratamiento antihipertensivo, índice de masa corporal y presencia de diabetes mellitus, definida según antecedentes de tratamiento hipoglicemiante o determinaciones sucesivas de glicemia en ayunas superior a $126 \mathrm{mg} / \mathrm{dL}$. 
El protocolo de este estudio contó con la aprobación del Comité de Ética Científica del Servicio de Salud Araucanía Sur. Todos los participantes firmaron voluntariamente un consentimiento informado escrito.

\section{Determinaciones bioquímicas}

Para las determinaciones de laboratorio se obtuvieron muestras de sangre venosa sin anticoagulante, previo ayuno de 12 horas. La concentración de colesterol total y triglicéridos fue determinada por métodos enzimático-colorimétricos; el colesterol HDL se midió previa precipitación selectiva de las LDL y VLDL con ácido fosfotúngstico e iones magnesio y posterior determinación por el método CHOD-PAP; la concentración de colesterol de las LDL fue calculada mediante la fórmula de Friedewald ${ }^{12}$. Las concentraciones séricas de glucosa y ácido úrico fueron determinadas mediante métodos enzimático colorimétricos.

\section{Genotipificación de la variante SNP-43 del gen CAPN10}

El ADN genómico fue extraído a partir de sangre total anticoagulada con EDTA mediante la técnica de precipitación salina descrita por Salazar et al. ${ }^{13}$. Para genotipificar la variante SNP-43 del gen CAPN10, se amplificó un fragmento de 175 pares de bases (bp) mediante la reacción en cadena de la polimerasa (PCR) en un termociclador MyCycler (Bio-Rad, California, EE.UU.), usando los partidores descritos previamente por Shima et al. ${ }^{14}$. El protocolo de PCR utilizado fue el siguiente: $200 \mathrm{nM}$ de cada primer, dNTPs $0,2 \mathrm{nM}$, cloruro de magnesio $\left(\mathrm{MgCl}_{2}\right) 2 \mathrm{mM}, 1 \mathrm{U}$ de Taq DNA Polimerasa (Fermentas, Lituania), $200 \mathrm{ng}$ de ADN y agua destilada estéril, para un volumen final de $50 \mu \mathrm{L}$. La mezcla de reacción se sometió a una denaturación inicial a $98^{\circ} \mathrm{C}$ durante 3 minutos y 30 ciclos compuestos por denaturación de 1 minuto a $95^{\circ} \mathrm{C}$, hibridación por 1 minuto a $60^{\circ} \mathrm{C}$ y una fase de extensión de 1 minuto a $72^{\circ} \mathrm{C}$, seguidos de una extensión final de 10 minutos a $72^{\circ} \mathrm{C}$. El producto amplificado se evaluó en gel de agarosa al $1,5 \%$ teñido con bromuro de etidio $(0.5 \mathrm{mg} / \mathrm{L})$ y visualizado en transiluminador UV.

Los productos amplificados fueron sometidos a digestión enzimática usando la endonucleasa NdeI (Fermentas, Lituania) mediante el siguiente protocolo: 10 $\mu \mathrm{L}$ de producto de PCR, $2.5 \mathrm{U}$ de NdeI, $2 \mu \mathrm{L}$ de buffer de reacción y agua destilada estéril hasta completar un volumen final de $20 \mu \mathrm{L}$. Esta mezcla de reacción fue incubada por 12 horas en baño termorregulado a $37^{\circ} \mathrm{C}$. Posteriormente, los fragmentos de restricción fueron evaluados mediante electroforesis en gel de agarosa al $3 \%$.

\section{Control de calidad de las determinaciones bioquímicas y moleculares}

La exactitud de las determinaciones bioquímicas fue controlada mediante la utilización de sueros comerciales normales y patológicos (Human, Alemania). La posibilidad de contaminación en los análisis moleculares fue excluida por la utilización de controles de reactivos en cada serie de amplificación. La correcta genotipificación del polimorfismo SNP-43 del gen CAPN10 fue confirmada mediante la repetición al azar del $10 \%$ de los análisis previamente realizados.

\section{Análisis estadístico}

El análisis de los datos obtenidos se realizó utilizando el programa Sigmaplot, versión 11.0 (Alemania). Todas las variables continuas se expresan como media \pm desviación estándar. Todos los parámetros bioquímicos mostraron distribución normal. La diferencia entre variables continuas se analizó mediante el test $t$ de Student. Para el análisis de las variables no continuas, así como distribución genotípica, frecuencia alélica y el equilibrio de Hardy - Weinberg se utilizó el test Chi-cuadrado $(\chi 2)$. La Odds ratio (OR) y su respectivo intervalo de confianza de $95 \%$, asociada al alelo mutado, fue también calculado. El nivel de significancia estadística considerado en este estudio fue $\mathrm{p}<0,05$.

\section{Resultados:}

\section{Análisis de las variables clínicas y de laboratorio}

Las características clínicas, antropométricas y de laboratorio de los sujetos en estudio son mostradas en la Tabla 1. Los individuos con EAC presentan valores elevados de índice de masa corporal (IMC) y una mayor prevalencia de factores de riesgo tradicionales para EAC, incluyendo consumo de tabaco, diabetes, hipertensión, hipercolesterolemia e historia familiar de EAC $(\mathrm{P}<0.001)$. Las concentraciones séricas de colesterol total, colesterol LDL, triglicéridos, glucosa y ácido úrico fueron más altas en los pacientes con EAC que en los controles $(\mathrm{P}<0.001)$. Además, los individuos con EAC presentaron niveles más bajos de HDL$\mathrm{C}(\mathrm{P}<0.001)$. Similarmente, se advirtió una diferencia significativa en los valores promedio de presión arterial sistólica $(\mathrm{P}<0.001)$ y diastólica $(\mathrm{P}=0.020)$ entre los grupos EAC y controles 


\begin{tabular}{|c|c|c|c|}
\hline & Casos (218) & Controles (194) & $\mathbf{P}^{*}$ \\
\hline Edad, a os & $62 \pm 10$ & $42 \pm 8$ & $<0.001$ \\
\hline Hombres, \% & 64 & 56 & 0.084 \\
\hline Diabetes, \% & 32 & 5 & $<0.001$ \\
\hline PAS, $\mathrm{mm} \mathrm{Hg}$ & $140 \pm 26$ & $125 \pm 23$ & $<0.001$ \\
\hline $\mathrm{PAD}, \mathrm{mm} \mathrm{Hg}$ & $80 \pm 18$ & $75 \pm 9$ & 0.020 \\
\hline IMC, kg/m2 & $28.2 \pm 4.5$ & $25.8 \pm 4.5$ & $<0.001$ \\
\hline Fumadores, \% & 64 & 41 & $<0.001$ \\
\hline Hipercolesterolemia, \% & 80 & 20 & $<0.001$ \\
\hline Hipertensi n, \% & 79 & 26 & $<0.001$ \\
\hline Angina, \% & 77 & 0 & $<0.001$ \\
\hline $\mathrm{IAM}, \%$ & 66 & 0 & $<0.001$ \\
\hline Historia Familiar de EAC, \% & 32 & 0 & $<0.001$ \\
\hline \multicolumn{4}{|c|}{$\mathrm{N}$ mero de vasos obstru dos, $\%$} \\
\hline 1 & $28 \%$ & 0 & --- \\
\hline 2 & $24 \%$ & 0 & --- \\
\hline 3 & $48 \%$ & 0 & --- \\
\hline Colesterol total, mg/dL & $214 \pm 51$ & $175 \pm 33$ & $<0.001$ \\
\hline $\mathrm{LDL}-\mathrm{C}, \mathrm{mg} / \mathrm{dL}$ & $127 \pm 42$ & $95 \pm 27$ & $<0.001$ \\
\hline $\mathrm{HDL}-\mathrm{C}, \mathrm{mg} / \mathrm{dL}$ & $32 \pm 8$ & $49 \pm 12$ & $<0.001$ \\
\hline Triglic ridos, mg/dL & $182 \pm 193$ & $114 \pm 86$ & $<0.001$ \\
\hline Glucosa, mg/dL & $113 \pm 42$ & $94 \pm 37$ & $<0.001$ \\
\hline cido rico, mg/dL & $5.6 \pm 1.6$ & $4.5 \pm 1.5$ & $<0.001$ \\
\hline
\end{tabular}

Tabla 1. Número de individuos en paréntesis; IAM; infarto agudo al miocardio; IMC, índice de masa corporal; EAC, enfermedad arterial coronaria; PAS, presión arterial sistólica; PAD, presión arterial diastólica; HDL-C, Colesterol de las HDL; LDL-C, Colesterol de las LDL. *Test t de Student o Chi-cuadrado.

\section{Genotipos y frecuencias alélicas}

La distribución de genotipos y frecuencia relativa de alelos para el polimorfismo SNP-43 del gen CAPN1O en individuos del grupo casos y controles, se muestran en la Tabla 2. La distribución de los genotipos están en concordancia con el equilibrio de Hardy-Weinberg en los controles $(\chi 2=1,2225 ; \mathrm{P}=\mathrm{NS})$, grupo casos $\left(\chi^{2}\right.$ $=0,0023 ; \mathrm{P}=\mathrm{NS})$ y para la totalidad de los pacientes en estudio $(\chi 2=0,7616 ; \mathrm{P}=\mathrm{NS})$. La frecuencia del genotipo A/A fue 5.9\% en el grupo casos y $5.3 \%$ en el grupo control. Sin embargo, no observamos diferencias significativas en la frecuencia genotípica y frecuencia alélica entre pacientes con EAC y controles $(\mathrm{P}=\mathrm{NS})$. La OR calculada para el genotipo homocigoto $\mathrm{A} / \mathrm{A}$ fue 1.17 (I.C. $95 \%, 0.50-2.72$ ) confirmando la ausencia de asociación. Por otro lado, dentro de los grupos estudiados no se observaron diferencias significativas entre las variables de laboratorio relativo a los genotipos $(\mathrm{P}=\mathrm{NS})$.

\section{Discusión:}

Diabetes mellitus tipo 2 y enfermedad arterial coronaria frecuentemente se manifiestan juntas, pudiendo existir una base genética común, pero pocos genes predisponentes a ambas patologías han sido identificados, siendo CAPN10 un gen candidato. Nuestros resultados muestran que no existe una asociación entre el polimorfismo SNP-43 del gen CAPN10 y enfermedad arterial coronaria. $\mathrm{Al}$ respecto, no existen reportes anteriores que den cuenta de una posible asociación entre el SNP-43, por sí solo, y esta patología.

Sin embargo, Goodarzi et al. han reportado que un haplotipo compuesto por los polimorfismos SNP-44, $-43,-56$ y -63 del gen $C A P N 10$, ha sido asociado, en varias poblaciones, con DMT2 $2^{10,15}$, y a aterosclerosis subclínica en población norteamericana ${ }^{11}$. Cabe destacar que en el estudio mencionado, se consideró como aterosclerosis subclínica al aumento del grosor 


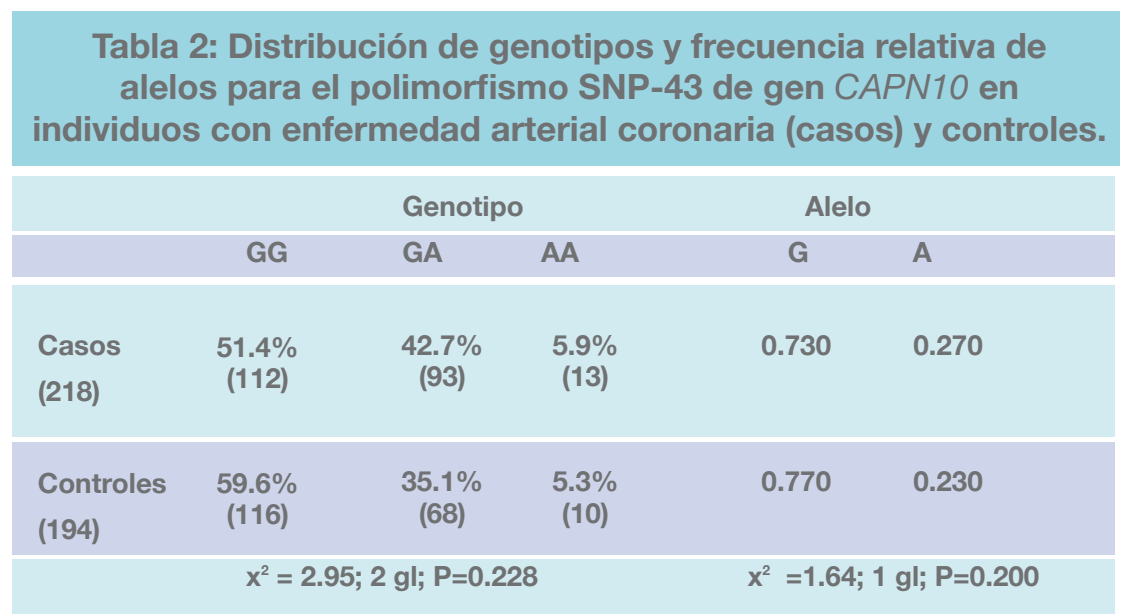

Tabla 2. Número de individuos en paréntesis; gl, grados de libertad.

mediointimal de la arteria carótida, medida indirecta utilizada como screening ${ }^{16}$, y tal aumento se asoció a un haplotipo integrado por el alelo mayor del SNP-43 (alelo G).

Por el contrario, el alelo menor (alelo A) fue asociado a un decremento del grosor mediointimal de la arteria carótida. Posteriormente el mismo autor, al replicar el estudio en una cohorte no hispana, encontró los hallazgos descritos previamente, con excepción de la disminución del grosor mediointimal de la carótida asociado al alelo $\mathrm{A}^{17}$. Estos únicos reportes que dan cuenta de una posible asociación entre variantes del gen $C A P N 10$ y aterosclerosis subclínica, sólo establecen una relación con un factor predictor de EAC en un estudio de cohorte de individuos sanos (cuyos padres padecen EAC), existiendo grandes diferencias con nuestro trabajo, ya que evaluamos mediante un estudio de casos y controles, la posible asociación entre el SNP-43 y EAC confirmada por angiografía. Así, no existe actualmente suficiente evidencia que pueda confirmar o rechazar una posible asociación, como factor de riesgo o protector, entre el polimorfismo en cuestión y EAC, considerando que los antecedentes existentes son controversiales.

Por otro lado, tampoco encontramos una asociación entre el SNP-43 y las variables clínicas y de laboratorio de los individuos en estudio (datos no mostrados), coincidiendo con lo encontrado en otros reportes ${ }^{9}$, aunque algunos estudios difieren de nuestros hallazgos. Carlsson et al., al estudiar este polimorfismo en una cohorte de obesos, encontró una asociación significativa entre el alelo mayor G del SNP-43 y concentraciones elevadas de triglicéridos ${ }^{18}$. Por otra parte, se ha encontrado asociación entre el genotipo G/G del SNP-43 tanto con concentraciones elevadas de colesterol total en suero ${ }^{19}$, así como con concentraciones más elevadas de glucosa a las 2 horas, posterior a una prueba de tolerancia a la glucosa, respecto a los genotipos G/A y $\mathrm{A} / \mathrm{A}^{20}$. Incluso, el genotipo $\mathrm{G} / \mathrm{G}$ se ha relacionado con hipertensión arterial en población asiática. ${ }^{21}$

Inicialmente, el SNP-43 del gen CAPN10 fue asociado a $\mathrm{DMT}^{21}$, hallazgo que ha sido confirmado por algunos metanálisis ${ }^{10,22}$ y desestimado por otros ${ }^{23}$, restando fuerza a la idea de que podría estar asociado a EAC. Esta doble postura puede deberse a un inadecuado poder estadístico de los análisis, diferencias raciales/étnicas en la frecuencia de los alelos, y especialmente a interacción gen-gen o gen-ambiente ${ }^{23}$, debido al carácter multifactorial de la DMT2 y EAC. Estos problemas pueden extrapolarse a las diferencias encontradas en la asociación de esta variante del gen CAPN10 con EAC, entre los pocos trabajos existentes respecto al tema. En lo que a nuestro trabajo se refiere, las frecuencias alélicas fueron muy similares a las publicadas en población europea (alrededor de 0.75 para el alelo mayor). Además, es necesario aumentar el tamaño muestral, para acrecentar el poder estadístico de nuestros resultados y estudiar los haplotipos descritos de CAPN10, debido a la amplia aceptación de la asociación DMT2 con el haplotipo SNP-43,-19, -63.

En suma, este polimorfismo se ha asociado a una mayor susceptibilidad de padecer DMT2, enfermedad ampliamente asociada a EAC, así como a otros factores de riesgo para EAC, como la hipertensión y concentraciones elevadas de glucosa y triglicéridos. Sin embargo, nuestros resultados sugieren que esta variante, por si sola, no es suficiente para incrementar el riesgo de padecer enfermedad coronaria en la población analizada. 


\section{Referencias}

1. HAFFNER SM. Coronary heart disease in patients with diabetes. N Engl J Med. 2000; 342: 1040-1042.

2. HAFFNER SM, LEHTO S, RONNEMAA T, PYORALA $\mathrm{K}$, LAAKSO M. Mortality from coronary heart disease in subjects with type 2 diabetes and in nondiabetic subjects with and without prior myocardial infarction. $\mathrm{N}$ Engl $\mathbf{J}$ Med. 1998; 339: 229-234.

3. KAO WH, HSUEH WC, RAINWATER DL, O'LEARY DH, IMUMORIN IG, STERN MP, et al. Family history of type 2 diabetes is associated with increased carotid artery intimal-medial thickness in Mexican Americans. Diabetes Care. 2005; 28: 1882-1889.

4. HORIKAWA Y, ODA N, COX NJ, LI X, ORHO-MELANDER $\mathrm{M}$, et al. Genetic variation in the gene encoding calpain-10 is associated with type 2 diabetes mellitus. Nat Genet. 2000; 26: 163-175.

5. MARSHALL C, HITMAN GA, PARTRIDGE CJ, CLARK A, MA H, SHEARER TR, et al. Evidence that an isoform of calpain-10 is a regulator of exocytosis in pancreatic beta-cells. Mol Endocrinol. 2005; 19: 213-224.

6. JOHNSON JD, HAN Z, OTANI K, YE H, ZHANG Y, WU $\mathrm{H}$, et al. RyR2 and calpain-10 delineate a novel apoptosis pathway in pancreatic islets. J Biol Chem. 2004; 279: 24794-24802.

7. MARQUEZ JL, PACHECO A, VALDES P, SALAZAR LA. Association between CAPN10 UCSNP-43 gene polymorphism and polycystic ovary syndrome in Chilean women. Clin Chim Acta. 2008; 398: 5-9.

8. EVANS JC, FRAYLING TM, CASSELL PG, SAKER PJ, HITMAN GA, WALKER M, et al. Studies of association between the gene for calpain-10 and type 2 diabetes mellitus in the United Kingdom. Am J Hum Genet. 2001; 69: 544-552.

9. GARANT MJ, KAO WH, BRANCATI F, CORESH J, RAMI TM, HANIS CL, et al. SNP43 of CAPN10 and the risk of type 2 Diabetes in African-Americans: the Atherosclerosis Risk in Communities Study. Diabetes. 2002; 51: 231-237.

10. TSUCHIYA T, SCHWARZ PE, BOSQUE-PLATA LD, GEOFFREY HAYES M, DINA C, FROGUEL $P$, et al. Association of the calpain-10 gene with type 2 diabetes in $\mathrm{Eu}$ ropeans: results of pooled and meta-analyses. Mol Genet Metab. 2006; 89: 174-184.

11. GOODARZI MO, TAYLOR KD, GUO X, QUINONES MJ, CUI J, LI Y, et al. Association of the diabetes gene calpain-10 with subclinical atherosclerosis: the MexicanAmerican Coronary Artery Disease Study. Diabetes. 2005; 54: 1228-1232.

12. FRIEDEWALD WT, LEVY RI, FREDRICKSON DS. Estimation of the concentration of low-density lipoprotein cholesterol in plasma, without use of the preparative ultracentrifuge. Clin Chem. 1972;18: 499-502.

13. SALAZAR LA, HIRATA MH, CAVALLI SA, MACHADO MO, HIRATA RD. Optimized procedure for DNA isolation from fresh and cryopreserved clotted human blood useful in clinical molecular testing. Clin Chem. 1998; 44: 1748-1750.

14. SHIMA Y, NAKANISHI K, ODAWARA M, KOBAYASHI T, OHTA H. Association of the SNP-19 genotype 22 in the calpain-10 gene with elevated body mass index and hemoglobin A1c levels in Japanese. Clin Chim Acta. 2003; 336: 89-96.

15. KANG ES, NAM M, KIM HJ, MYOUNG SM, RHEE Y, AHN CW, et al. Haplotype combination of Calpain-10 gene polymorphism is associated with metabolic syndrome in type 2 diabetes. Diabetes Res Clin Pract. 2006; 73: 268 275 .

16. NGUYEN-THANH HT, BENZAQUEN BS. Screening for subclinical coronary artery disease measuring carotid intima media thickness. Am J Cardiol. 2009; 104: 1383-1388.

17. GOODARZI MO, TAYLOR KD, JONES MR, FANG B, GUO X, XIANG AH, et al. Replication of calpain-10 genetic association with carotid intima-media thickness. Atherosclerosis. 2009

18. CARLSSON E, FREDRIKSSON J, GROOP L, RIDDERSTRALE M. Variation in the calpain-10 gene is associated with elevated triglyceride levels and reduced adipose tissue messenger ribonucleic acid expression in obese Swedish subjects. J Clin Endocrinol Metab. 2004; 89: 3601-3605.

19. DAIMON M, OIZUMI T, SAITOH T, KAMEDA W, YAMAGUCHI H, OHNUMA H, et al. Calpain 10 gene polymorphisms are related, not to type 2 diabetes, but to increased serum cholesterol in Japanese. Diabetes Res Clin Pract. 2002; 56: 147-152.

20. LYNN S, EVANS JC, WHITE C, FRAYLING TM, HATTERSLEY AT, TURNBULL DM, et al. Variation in the calpain-10 gene affects blood glucose levels in the British population. Diabetes. 2002; 51: 247-250.

21. CHEN SF, LU XF, YAN WL, HUANG JF, GU DF. Variations in the calpain-10 gene are associated with the risk of type 2 diabetes and hypertension in northern Han Chinese population. Chin Med J (Engl). 2007; 120: 2218-2223.

22. WEEDON MN, SCHWARZ PE, HORIKAWA Y, IWASAKI N, ILLIG T, HOLLE R, et al. Meta-analysis and a large association study confirm a role for calpain-10 variation in type 2 diabetes susceptibility. Am J Hum Genet. 2003; 73: 1208-1212.

23. SONG Y, NIU T, MANSON JE, KWIATKOWSKI DJ, LIU S. Are variants in the CAPN10 gene related to risk of type 2 diabetes? A quantitative assessment of population and family-based association studies. Am J Hum Genet. 2004; 74: 208-222. 